\title{
TUNNELLING OF DIRECT AND INDIRECT EXCITONS IN SLIGHTLY ASYMMETRIC DOUBLE QUANTUM WELLS
}

\author{
D.A. Mazurenko, A.V. Akimov, E.S. Moskalenko, A.L. Zhmodikov, \\ A.A. KaPLYANSKII
}

A.F. Ioffe Physical-Technical Institute, Russian Academy of Sciences

26 Polytechnicheskaya Str., 194021, St. Petersburg, Russia

L.J. Challis, T.S. Cheng and C.T. Foxon

Physics Department, Nottingham University

University Park NG7 2RD, Nottingham, U.K.

We report the first studies of luminescence spectra from asymmetric double quantum wells of GaAs with widths around $100 \AA$ differing by only one $(2.8 \AA)$ or two monolayers. Studies of the position and intensity of the direct and indirect exciton lines suggest the existence of acoustic phonon-assisted tunnelling between exciton states separated by a few meV. At temperatures $10-20 \mathrm{~K}$ the electron tunnelling process is fast enough to maintain thermal equilibrium between these direct and indirect excitons which are connected with holes in the same quantum well.

PACS numbers: 73.20.Dx, 63.20.Kr

In GaAs/AlGaAs double quantum wells (DQWs) electron and hole tunnelling through the thin barrier have been intensively studied by both $\mathrm{cw}$ and time-resolved luminescence techniques and it is clear that the tunnelling processes are significantly modified by excitonic effects [1]. Much of this work was carried out on asymmetric DQWs in which the ground state energy differences in the two wells were appreciably more than $1 \mathrm{meV}$ for both electrons and holes. For smaller energy differences, it has not yet been possible to separate the non-resonant tunnelling associated with acoustic phonons from that caused by elastic scattering from impurities and interface defects followed by phonon relaxation. For simplicity therefore we refer to the observed process as phonon-assisted tunnelling in the expectation that in our high quality samples this will turn out to be the stronger of the two. A theoretical analysis of these processes has been carried out $[2,3]$ and a direct observation of acoustic phonon-assisted tunnelling in double barrier resonant tunnelling structures has been recently obtained using phonon pulses [4]. Detailed information on all these relaxation and tunnelling processes would be of value in the development of semiconductor nanostructures as phonon spectrometers and generators at frequencies up to $1 \mathrm{THz}$ and above [2-5].

In the present paper we are studying the $\mathrm{cw}$ photoluminescence from slightly asymmetric DQWs, in which the widths of the coupled QWs differ by only 1 or 2 monolayers (MLs). The paper is aimed particularly at extracting information on 
the population of different exciton states of DQW. Now in these slightly asymmetric systems, the difference in excitonic transition energy in the two QWs of only a few $\mathrm{meV}$ is smaller than the exciton binding energy $E_{\mathrm{B}}$. So a process in which luminescence from the narrower well creates electron-hole pairs and hence excitons in the wider well - a process which is important in more asymmetric DQWs - can no longer occur.

Our structures were grown by MBE on $0.4 \mathrm{~mm}$ thick semiinsulating GaAs substrates. To apply external electric field a semitransparent film was evaporated above the DQWs to form a Schottky contact and an indium film was deposited on the back side of the substrate.

In the present contribution we concentrate on the results obtained with the DQW whose parameters are shown in Fig. 1. Figure 1 also shows schematically the direct and indirect exciton transitions in the DQW. Direct excitons (DX) originate from the electrons (e) and heavy holes (h) located in the same QW (narrow DXN or wide - DXW), while for indirect excitons (IX) the e and $h$ are in different QWs - IXN (IXW) when $h$ is in the narrow (wide) QW.

The luminescence spectra excited by a He-Ne laser beam $(\lambda=730 \mathrm{~nm})$ at $20 \mathrm{~K}$ consist of four narrow ( $\leq 1 \mathrm{meV}$ ) lines whose energies correspond to the recombination of the DX and IX excitons. These are shown in Fig. 2a for several values of applied voltage $V$. The position of the dominant line 1 , which for low $V$ corresponds to DXW, shifts to lower energy as $V$ increases and, for $V>2 \mathrm{~V}$, a second line 2 appears on the high energy side, increases in intensity and approaches an energy equal to the initial energy of line 1 . Line 4 which position at low $V$ corresponds to DXN shifts to higher energies with $V$ and another line, 3, appears whose energy approaches the initial energy of line 4.

Two of the four observed lines are attributable to DX and the other two to IX. The quadratic Stark effect is negligible for the fields applied so that the energies of DX are essentially independent of $V$. However the energy of IX depends linearly on $V$ so that they can be conveniently moved relative to those of DX. Coherent tunnelling of "excitonic" electrons near DXW(N)/IXW(N) resonance leads to anticrossing and so to splitting between the symmetric and antisymmetric combinations of the DX and IX eigenstates [1]. The effect of this anticrossing can be seen in Fig. 2 and a detailed analysis of the peak position of lines 1-4 as a function of $V$ gives values for the resonant tunnelling splitting and the IX binding energy of $\delta=1.33 \mathrm{meV}$ and $E_{\mathrm{IX}}=3.3 \mathrm{meV}$, respectively which are both in reasonable agreement with those of earlier studies [1].

We next discuss the relative intensities of the exciton luminescence lines which are governed by competing between recombination and phonon-assisted exciton tunnelling from one $\mathrm{QW}$ to the other. If the tunnelling rate $\left(\omega_{\mathrm{f}}^{i k}\right)$ is much higher than the exciton recombination rate $\left(\omega_{\mathrm{R}}^{i}, \omega_{\mathrm{R}}^{k}\right)$ then excitons $i, k$ with energies $E_{i}, E_{k}$ will be in thermal equilibrium and relative intensities of pairs of exciton lines can be expressed as $I_{i} / I_{k}=\left(\omega_{\mathrm{R}}^{i} / \omega_{\mathrm{R}}^{k}\right) \exp \left[-\left(E_{i}-E_{k}\right) / k T\right]$. The values (Fig. 3) of $I_{i} / I_{k}$ as a function of $E_{i}-E_{k}$ were calculated using the well-known [1] expressions for the electric field dependence of $\omega_{\mathrm{R}}^{i}, \omega_{\mathrm{R}}^{k}$ and $E_{i}-E_{k}$ under thermal equilibrium conditions and Fig. 3 shows that these are in good agreement with the experimental data for DX/IX exciton pairs $i, k$ in which the hole is in the same 


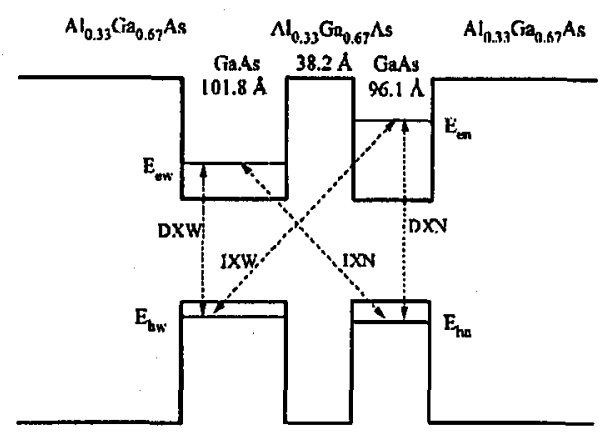

Fig. 1. The band diagram and the scheme of the exciton transitions in the DQW. The vertical dashed lines show direct excitons in wide (DXW) and narrow (DXN) QWs and the slanted lines show indirect excitons in which a heavy hole is located in a wide (IXW) or narrow (IXN) QW.
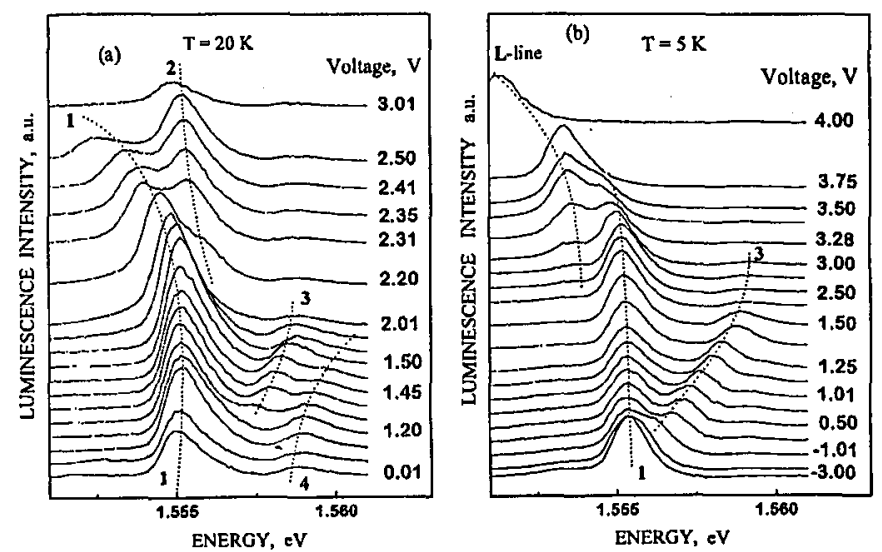

Fig. 2. Luminescence spectrum of DQW measured at (a) $20 \mathrm{~K}$ and (b) $5 \mathrm{~K}$ for different applied voltages. The dotted lines are drawn for the eye to follow the peak position of the exciton lines.

QW so that thermal equilibrium can be established by electron tunnelling DXN $\leftrightarrow$ IXN, DXW $\leftrightarrow$ IXW. However the interaction between DX/IX pairs in which the electron is in the same QW is much weaker since it involves hole tunnelling which is a very much slower process and no equilibrium is established between corresponding levels. It is seen from the spectra in Fig. $2 \mathrm{~b}$, at $T=5 \mathrm{~K}$, when only two lines 1 and 3 are detectable. Indeed at $V=1.4 \mathrm{~V}$ (where lines 1 and 3 are mostly DX states) $I_{3} / I_{1}=N_{3} / N_{1}=0.46$ which is very much bigger than the ratio $N_{3} / N_{1}=1.3 \times 10^{-4}$ calculated for the Boltzmann distribution showing that the excitons 1 and 3 are far from thermal equilibrium at $5 \mathrm{~K}$ and this remains true at $20 \mathrm{~K}$.

We conclude that the exciton pairs $1 / 2$ and $3 / 4$ are each in internal thermal equilibrium but that there is no equilibrium between them. The equilibrium within 


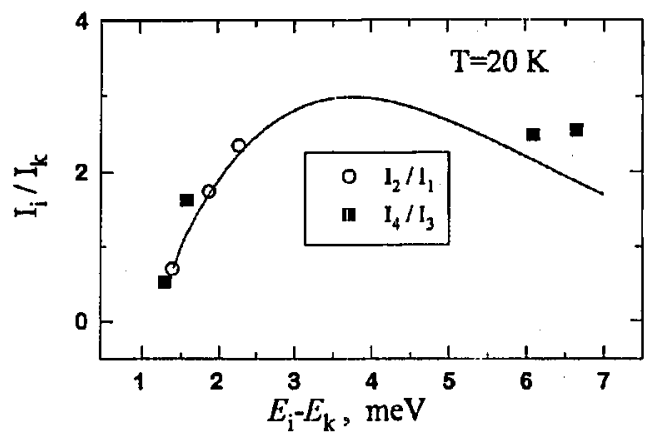

Fig. 3. The measured (symbols) and calculated (solid curves) ratios $I_{i} / I_{k}$ of the exciton line intensities as a function of the energy separation between the $i$ - and $k$-lines for $T=20 \mathrm{~K}$.

these pairs is maintained by phonon-assisted electron tunnelling and the existence of the equilibrium implies that this process is much faster than the radiative decay process $\omega_{\mathrm{t}}^{\mathrm{e}} \gg \omega_{\mathrm{R}}^{\mathrm{DX}} \sim 10^{9} \mathrm{~s}^{-1}$. Coupling between pairs $1 / 2$ and $3 / 4$ takes place by hole tunnelling and the lack of equilibrium in this case implies that this rate is slower than the radiative decay rate, $\omega_{\mathrm{t}}^{\mathrm{h}}<\omega_{\mathrm{R}}^{\mathrm{DX}} \sim 10^{9} \mathrm{~s}^{-1}$. The large difference between the phonon-assisted electron and hole tunnelling rates is consistent with earlier results [1] for strongly asymmetric DQWs and attributable to the large hole mass and so weaker penetration into the barrier of the hole wave function.

Finally we discuss the origin of the new not reported earlier long wavelength line which we refer as L-line, which appears at $T=5 \mathrm{~K}$ at $V>3 \mathrm{~V}$ (see Fig. $2 \mathrm{~b}$ ). We attribute this to a localized indirect bound exciton since the size of its red-shift relative to IXW is close to the binding energy of excitons in GaAs QWs [6]. Line $\mathrm{L}$ disappears for $T>10 \mathrm{~K}$ while line 1 remains and this is consistent with the dissociation of localized excitons (which have only a small density of states) into free excitons with increasing temperature.

We gratefully acknowledge financial support from the Russian Foundation for Fundamental Studies (No. 92-02-16952a) and the European Commission (INTAS94-395).

\section{References}

[1] For comprehensive review see J. Shah, in: Optics of Semiconductor Nanostructures, Eds. F. Henneberger, S. Schmitt-Rink, E.O. Gobel, Akademie Verlag, Berlin 1993, p. 149.

[2] F.T. Vasko, O.G. Balev, P. Vasilopoulos, Phys. Rev. B 47, 16433 (1993).

[3] V.I. Kozub, A.M. Rudin, Phys. Rev. B 49, 5710 (1994).

[4] F.F. Ouali, N.N. Zinov'ev, L.J. Challis, F.W. Sheard, M. Henini, D.P. Steenston, K.R. Strickland, Phys. Rev. Lett. 75, 308 (1995).

[5] A.V. Akimov, E.S. Moskalenko, L.J. Challis, A.A. Kaplyanskii, Physica B 219, 9 (1996).

[6] D.C. Reynolds, K.R. Evans, K.G. Merkel, C.E. Stutz, P.W. Yu, Phys. Rev. B 43, 9087 (1991). 